\title{
Protein-Synthetic Machinery at Postsynaptic Sites During Synaptogenesis: A Quantitative Study of the Association Between Polyribosomes and Developing Synapses
}

\author{
Oswald Steward and Paula M. Falk \\ Departments of Neurosurgery and Physiology, University of Virginia School of Medicine, \\ Charlottesville, Virginia 22908
}

Previous studies have revealed dramatic accumulations of polyribosomes under growing spine synapses, suggesting a critical role for protein synthesis at the postsynaptic site during synaptogenesis. The present study quantitatively analyzes the distribution of polyribosomes under synapses during developmental synaptogenesis in the rat's dentate gyrus. The middle molecular layer of the suprapyramidal blade of the dentate gyrus was examined electron-microscopically at 1, 4, 7, 10, 15, 20, and $28 \mathrm{~d}$ of age. At each age, we evaluated (1) synapse density (the number of synapses $/ 100 \mu \mathrm{m}^{2}$ of neuropil), (2) the width of the molecular layer, (3) the proportion of spine synapses with underlying polyribosomes, and (4) the number of polyribosomecontaining synapses/1000 $\mu \mathrm{m}^{2}$ of neuropil. From the first two measures, an estimate was obtained of the total extent of synaptogenesis, taking into account both the increase in synapse density and the increase in total area of neuropil.

At $1 \mathrm{~d}$ of age, very few synapses were found in the molecular layer of the dentate gyrus, and those that were present were quite immature in appearance. Synapse density increased about 140-fold between 1 and $28 \mathrm{~d}$ of age, from an average of 0.36 synapses $/ 100 \mu \mathrm{m}^{2}$ at $1 \mathrm{~d}$ of age to 49 synapses $/ 100 \mu \mathrm{m}^{2}$ at $28 \mathrm{~d}$ of age. An inverse relationship was found between synapse density and the proportion of synapses with polyribosomes. Between 1 and $7 \mathrm{~d}$ of age, about $60 \%$ of the spine synapses had one or more polyribosomes under the spine base. Thereafter, the proportion of spines with polyribosomes decreased as synapse density increased. Similarly, the proportion of shaft synapses with underlying polyribosomes was greatest between 1 and $7 \mathrm{~d}$ postnatal, and decreased thereafter. While the proportion of synapses with polyribosomes was greatest between 1 and $7 \mathrm{~d}$, the actual number of polyribosome-containing synapses/ $1000 \mu \mathrm{m}^{2}$ of neuropil was negligible at $1 \mathrm{~d}$, increased to a peak at $7 \mathrm{~d}$ of age, and then decreased as synapse density increased. Qualitatively, the most dramatic accumulations of polyribosomes were also found at $7 \mathrm{~d}$ of age.

We conclude that spine-associated polyribosomes represent a structural specialization of dendrites at sites of synapse construction and as such may represent a marker for growing syn-

\footnotetext{
Received Apr. 11, 1985; revised Aug. 23, 1985; accepted Aug. 26, 1985.

Thanks to S. L. Vinsant and F. L. Snavely for their excellent technical help in the early stages of the electron microscopic analysis. Thanks also to R. A. Ogle for help with the illustrations, and to M. P. Janssen for secretarial help. This work was supported by National Institutes of Health Grant NS 12333 to O.S. O.S. was the recipient of a Research Career Development Award (NS 00325) during the time that much of this work was undertaken. A preliminary account of some of these results has been published (Steward and Falk, 1985).

Correspondence should be addressed to Oswald Steward, Departments of Neurosurgery and Physiology, University of Virginia School of Medicine, Charlottesville, VA 22908.

Copyright (C) 1986 Society for Neuroscience $0270-6474 / 86 / 020412-12 \$ 02.00 / 0$
}

apses. We propose that these elements produce protein(s) that are critically involved in the formation of the synaptic contact.

Despite the obvious importance of synaptogenesis in neural development, relatively little is known about exactly what cellular and molecular processes are involved in the making of a synapse. Synapse formation clearly requires the elaboration of several types of structural specializations, including axons, dendrites, pre- and postsynaptic contact regions, and, in many neurons, dendritic spines; however, most of our knowledge about the cellular mechanisms of neuron growth pertains to growing axons. An area of particular interest about which relatively little is known involves the construction of the portion of the postsynaptic cell that is specialized for the receipt of the synapse. Neurons of the CNS receive most of their synaptic contacts upon postsynaptic membrane specializations (the postsynaptic density). These membrane specializations are thought to represent the active zone of the synapse, and presumably contain a variety of molecules involved in synaptic function, including neurotransmitter receptors, ion channels, molecules to immobilize the membrane proteins to keep them localized at the synaptic site, regulatory enzymes such as kinases and adenylate cyclase, molecules to define the chemical identity (specificity) of the contact, and adhesion molecules (for studies of the composition of the postsynaptic density, see Carlin et al., 1981; Cohen et al., 1977; Feit et al., 1977; Grab et al., 1981a, b; Kelly and Cotman, 1978). Furthermore, many neurons in the vertebrate CNS elaborate microspecializations of dendritic form at each site of synaptic contact (dendritic spines).

The remarkable feature of many CNS neurons is that they elaborate a multitude of specializations for synaptic contact all along their dendritic processes, sometimes with different types of contacts on different portions of the dendritic tree. For example, Purkinje cells of the cerebellum construct tens of thousands of contact sites along their dendrites, some specialized for the receipt of parallel fibers and others for the receipt of climbing fibers. The situation for pyramidal cells of the cerebral cortex or the hippocampus is even more complex, since these cells may receive many different afferent types, each terminating on a particular portion of the dendritic tree. In these cases, not only must the neuron construct thousands of synaptic sites; it must somehow organize its receptive surface so as to accommodate different types of synapses (presumably with different requirements for receptors, etc.) at different locations. How such microspecializations are constructed remains largely a mystery, although until recently it was generally assumed that the materials for the contact site were synthesized in the neuronal cell body and delivered to the synaptic site by some sort of targeted transport system.

Our recent discovery of protein-synthetic machinery (poly- 
ribosomes) at the base of dendritic spines of CNS neurons (see Steward, 1983b; Steward and Levy, 1982) has provided a new way of thinking about how synapses might be constructed and maintained. Specifically, regulation of postsynaptic contact regions could involve a local synthesis of some protein(s) at the synaptic site, rather than depending entirely on transport of material synthesized in the cell body. In this regard, it is of particular interest that the incidence of polyribosomes under spines increases dramatically during synapse growth induced, in mature animals, by denervating lesions (Steward, 1983a). These data led us to propose that local synthesis at the postsynaptic site might be particularly important during synapse growth (Steward, 1983a, b; Steward and Fass, 1983).

While the studies cited above clearly suggested a role for the polyribosomes in lesion-induced reinnervation, the precise nature of their role remained unclear. Synapse replacement following lesions involves processes that may not be important during normal development, such as those involved in the degradation of old sites of contact left vacant by the removal of normal innervation. It was therefore of interest to evaluate whether polyribosomes were also a prominent feature of growing synapses in the developmental period. Furthermore, it was of interest to determine when, in the synaptogenic period, the polyribosomes were most prevalent. Particularly interesting was the question of whether polyribosomes were most prominent at the time of initial contact formation, or during some later phase of the differentiation of the contact zone. In the present study, the relationship between polyribosomes and developing synapses is evaluated in the dentate gyrus of the rat, using quantitative electron-microscopic techniques.

The dentate gyrus offers several advantages for studies of developmental synaptogenesis, owing to its synaptology and relatively late development. The dendrites of the granule cells and their associated afferents are found in a neuropil layer (the molecular layer), where each of the major afferents terminates in a discrete lamina. Throughout the neuropil, most of the synaptic connections terminate on dendritic spines with characteristic mushroom or lollipop shape in mature animals (Steward and Vinsant, 1983). Most of the differentiation of granule cells occurs postnatally. Approximately $90 \%$ of the granule cells undergo their final mitosis after birth (Bayer, 1980; Schlessinger et al., 1975). From birth until about $20 \mathrm{~d}$ of age, granule cells elaborate their dendritic processes, and come to receive afferent inncrvation (Fricke and Cowan, 1977; Loy et al., 1977; for a review, see Cowan et al., 1980). Thus, from birth until about $20 \mathrm{~d}$ of age, the dentate gyrus is a site of formation of an enormous number of synaptic connections. Early in this period, virtually any synapse observed must be either in the process of formation, or newly formed. This is an optimal setting for studying cellular specializations associated with growing synapses.

We report that developing synapses have even more dramatic accumulations of polyribosomes than synapses growing in response to lesions; the number of polyribosomes under synapses decreases with synapse maturation. A preliminary account of some of these results has been published (Steward and Falk, 1985).

\section{Materials and Methods}

Sprague-Dawley-derived rats of both sexes were prepared for EM at 1 , $4,7,10,15,20$, and $28 \mathrm{~d}$ of age (two animals at each interval, except for 1,4 , and $15 \mathrm{~d}$, when three animals were analyzed). The day of birth is considered day 0 . The animals were deeply anesthetized with sodium pentobarbital and perfused transcardially with mixed aldehyde fixatives warmed to $30-37^{\circ} \mathrm{C}$. Most of the animals were perfused with the same fixative that we routinely use with mature animals ( $2 \%$ paraformaldehyde $/ 2 \%$ glutaraldehyde in $0.13 \mathrm{M}$ cacodylate buffer, $\mathrm{pH} 7.2$ ), although at both 7 and $10 \mathrm{~d}$ of age, one animal was perfused with $1 \%$ paraformaldehyde $/ 1.5 \%$ glutaraldehyde in $0.13 \mathrm{~m}$ cacodylate buffer. The brains were removed, and postfixed in the perfusion solution for about $1 \mathrm{hr}$, then sectioned on an Oxford Vibratome. Sample blocks of the dentate gyrus from approximately one-quarter the way along the septotemporal axis of the dentate gyrus were dissected freehand, osmicated, and embedded in plastic using routine procedures (Steward and Vinsant, 1983). Thin-sections were cut perpendicular to the layer of granule cell bodies in order to obtain sections that extended from the cell body.layer to the zone containing the tips of the granule cell dendrites (the outer molecular layer). Both suprapyramidal and infrapyramidal blades of the dentate gyrus were evaluated qualitatively, and representative photomicrographs were taken at $10,000 \times$.

For the quantitative analyses, a sample of $42-45$ photographs were taken in the middle molecular layer of the suprapyramidal blade of the dentate gyrus in each animal (initial magnification, 10,000 $\times$; final magnification, 27,000 $\times$ ). For all animals, except those prepared at 1 and 4 $\mathrm{d}$ of age, the sampling procedure was similar to that described in Steward and Levy (1982). The middle molecular layer was scanned for spines that were visible in their entirety (from their point of emergence on the parent dendrite to the region of synaptic contact), and photomicrographs, centered on such identified spines, were taken. Each photograph represented a cross-sectional area of about $55-60 \mu \mathrm{m}^{2}$, providing an average sample area of $2321 \mu \mathrm{m}^{2}$ of neuropil in each animal (range, $2103-2514 \mu \mathrm{m}^{2}$ ). For the animals prepared at 1 and $4 \mathrm{~d}$ of age, synapses were rare, and those that were present were often found in clusters; thus for these animals, rows of photographs were taken from the middle third of the molecular layer in order to sample the same total area of neuropil as above.

From these collections of photomicrographs, we evaluated the number of spine and shaft synapses. Synapses were defined as appositions between a presynaptic process with at least three synaptic vesicles and a postsynaptic element with a definable postsynaptic specialization (see Steward and Vinsant, 1983). In the molecular layer of mature rats, the vast majority of synapses are on well-developed spines (Stcward and Vinsant, 1983). In the very young animals, however, very few welldeveloped spines were observed. Rather, synapses tended to be found on very stubby protrusions from the dendrite. It seems likely that these stubby protrusions are the precursors of spines, since they represent the predominant form in the young animals, and in mature animals most synapses are on spines. In addition, these stubby protrusions usually contain flocculent material resembling that found in mature spines. Thus, any synapse that was associated with even a slight irregularity of the smooth profile of the dendritic shaft and contained such flocculent material was scored as a spine synapse. Synapses not associated with irregularities of the dendritic contour were scored as shaft synapses. After determining the number of synapses in each collection of photomicrographs, the total cross-sectional area covered by the photomicrographs was determined. From these values, the numbcr of spinc and shaft synapses $/ 100 \mu \mathrm{m}^{2}$ was determined for each animal. We also measured the width of the molecular layer of the dentate gyrus (from the top of the granule cell layer to the hippocampal fissure) in order to provide an estimate of the increase in the area of neuropil. These values, together with the estimates of synapse density, could then provide an estimate of the total extent of synaptogenesis in the molecular layer across the developmental period.

For the analysis of the proportion of spine synapses with underlying polyribosomes, we determined the relative incidence of polyribosomes under "identified spine bases." Following conventions that we had established previously (Steward, 1983a; Steward and Levy, 1982), "identified spine bases" refer to intersections between a main dendrite and a spine neck. Spine necks were identified as such on the basis of (1) being continuous with a spine head that received a presynaptic contact upon an area of membrane specialization (the entire profile of the spine must be visible in the section) or (2) containing a well-defined spine apparatus. At all ages-but particularly in the young animals-most of the spines were included in the sample on the basis of criterion (1). A spine was considered positive for polyribosomes if the clusters were found under the base of the spine. However, if microtubules or other organelles were found between the polyribosomes and the spine base, the polyribosomes were considered to lie within the dendrite proper, and the spine was scored as polyribosome-free.

Polyribosomes were defined as collections of three or more particles of the size and electron density of ribosomes, with an interparticle spacing typical of polyribosomes. It is important to note that this is a morphological identification, which is not unequivocal. Nevertheless, because of their uniform size, the particles wcrc clcarly distinguishable from other similar electron-dense granules, including glycogen; if they 
are not polyribosomes, they are an unidentified cytoplasmic component. Since previous studies have shown that the particles are sensitive to ribonuclease treatment (Steward, 1983b), their identification as polyribosomes seems reasonably secure.

We also counted the number of polyribosome-containing spine heads that were not connected with a dendritic shaft in the plane of section, and the number of shaft synapses with underlying polyribosomes. From these, we calculated the number of polyribosome-positive synapses/ $1000 \mu \mathrm{m}^{2}$ of neuropil. The values obtained for synapse density, the relative incidence of polyribosomes under spines, and the number of polyribosome-containing synapses/1000 $\mu \mathrm{m}^{2}$ of neuropil were compared with values for adult animals obtained from material prepared for other studies (Steward, 1983a; Steward and Levy, 1982; Steward and Vinsant, 1983).

\section{Results}

\section{Qualitative observations}

Our qualitative evaluations focused on the question of when, in the process of synapse formation, the polyribosomes were most evident. Some of the issues were whether polyribosomes were present at future synaptic sites prior to the differentiation of morphologically identifiable synaptic junctions, and whether the polyribosomes were most prominent under the youngestappcaring synapses. Thus, the distribution of polyribosomes under noninnervated synaptic specializations and under synaptic specializations apposed to apparent growth cones was of particular interest. Also of interest was whether the number of polyribosomes increased during the maturation of the contact region.

At $1 \mathrm{~d}$ of age, the gradient of differentiation in the dentate gyrus was particularly apparent. In the suprapyramidal blade, there was already a discernible cell layer and thin molecular layer. In contrast, the infrapyramidal blade was much less mature, and was characterized by a loosely organized collection of cells, with no evidence of a molecular layer. Within the molecular layer of the suprapyramidal blade, relatively few synaptic contacts were found. Rather, the layer contained numerous microtubule-containing processes, and presumed growth cones (see Fig. 1). Some of the microtubule-containing processes were clearly dendrites, since they could be seen to emerge directly from the neuronal somata of the granule cell layer. These contained accumulations of polyribosomes. Other processes were less easy to identify, since they contained neither polyribosomes nor synaptic vesicles. Many of these may be axons. In addition to the microtubule-containing profiles, there were numerous processes filled with a filamentous material with few other intracellular organelles (Fig. 1A). These appeared to be identical to the types of growth cones that have been identified in a number of brain regions (Hinds and Hinds, 1976; Skoff and Hamburger, 1974; Tennyson, 1970; Vaughn and Sims, 1978). Most of these could not be identified as axonal or dendritic, except when they could be seen in continuity with ribosome-containing processes or when they emerged from processes with identifiable synaptic relations with other elements. In addition, some processes exhibited bulbous enlargements with endoplasmic reticulum (see Fig. 1, $A$ and $B$ ). These processes resembled the growth cones described by Del Cerro and Snider (1968) and Kawana et al. (1971). There were also numerous vesicle-filled mounds, similar to those frequently found on growth cones in culture (Pfenninger and Rees, 1976).

The few synapses present at $1 \mathrm{~d}$ of age were typically found on stubby protrusions from the dendrites (see Fig. 1C). Because there are few shaft synapses in the mature dentate gyrus, and because most of these stubby protrusions contained flocculent material similar to that found in mature spines, we have identified these as very immature spine synapses for quantitative purposes. Many of these presumed spines were marked by accumulations of polyribosomes, although the collections of poly- ribosomes were not as dramatic as at later ages (see below). There was a tendency for the synapses to occur in clusterssome photographic fields had several synapses, while the vast majority had none.

In addition to the synapses, there were unapposed postsynaptic densities, some of which had underlying accumulations of polyribosomes (see Fig. 1, $A$ and $D$ ). In addition, specializations resembling postsynaptic densities were sometimes found at sites of contact between two dendritic processes (identified by the presence of polyribosomes). These dendrodendritic contacts were also present in $4 \mathrm{~d}$ old animals (see Fig. $2 D$ ), but were not apparent in animals more than $4 \mathrm{~d}$ of age. Some caution must be exercised in identifying all polyribosome-containing profiles as dendrites, since one profile was observed which contained vesicles of the size of synaptic vesicles, yet polyribosomes were present on the presynaptic side. It could not be determined whether this unique profile was a dendrodendritic chemical synapse or an axodendritic synapse, where the presynaptic member contained polyribosomes.

At $4 \mathrm{~d}$ of age, the infrapyramidal blade of the dentate gyrus still did not exhibit a definable molecular layer. The molecular layer of the suprapyramidal blade contained dendrites (identified by the presence of ribosomes), microtubule-containing processes that werc probably axons, and presumed growth cones (see Fig. 2). At this age, there were fewer of the vesicle-filled mounds than at $1 \mathrm{~d}$ of age, although the presumed growth cones filled with filamentous material, and the processes filled with endoplasmic reticulum were still quite common (see Fig. $2 A$ ). The number of synapses was obviously considerably higher than at $1 \mathrm{~d}$ of age, although the distribution was still patchy; again, several synapses were often found in a given photographic field, while other fields contained none. The synapses that were present at $4 \mathrm{~d}$ of age were more often on profiles that clearly protruded from the dendritic shaft (see Fig. 2); the identification of such profiles as spines was thus less ambiguous than was the case at $1 \mathrm{~d}$ of age. A few profiles could be found in which the spine had a shape similar to that in adult animals, with a thin neck and enlarged head (Fig. 2C).

While synapses were more numerous than at $1 \mathrm{~d}$ of age, most still appeared quite immature, in that the presynaptic element often had few vesicles, and the vesicles that were present were often not accumulated near the contact region (Fig. $2 E$ ). Some apparent spines were also apposed to the enlarged filamentous profiles thought to represent growth cones (Fig. 2A). Vesicles larger than typical synaptic vesicles were also common (Fig. $2 E$ ). In addition, the postsynaptic membrane specialization was often quite thin. All of these features are characteristic of growing synapses in other brain regions (Bloom, 1972; Foelix and Oppenheim, 1974; Hinds and Hinds, 1976; Vaughn and Sims, 1978). Although a few profiles could be found that resembled poorly developed spines, without identifiable presynaptic contacts, these were not common. Unapposed postsynaptic densities were also found, but these were less common than at $1 \mathrm{~d}$ of age. The accumulations of polyribosomes under synapses tended to be more dramatic than at $1 \mathrm{~d}$ of age, in that more rosettes were present per synapse (see Fig. $2, A, B, F$, and $G$ ); nevertheless, the accumulations were still not as dramatic as at $7 \mathrm{~d}$ of age. While more polyribosomes were also present in the dendrite proper than with mature animals, the majority of the dendritic polyribosomes appeared to lie under spines or mounds in the dendrite, resembling the bases of spines (see Fig. 2B). Thus, as is also true in mature animals (Steward and Levy, 1982), the localization of polyribosomes under spines appeared to be selective. No quantitative evaluation of the degree of this selectivity was undertaken, however.

At $7 \mathrm{~d}$ of age, both supra- and infrapyramidal blades possessed a definable molecular layer. The infrapyramidal blade was characterized by considerable extracellular space, with scattered ax- 

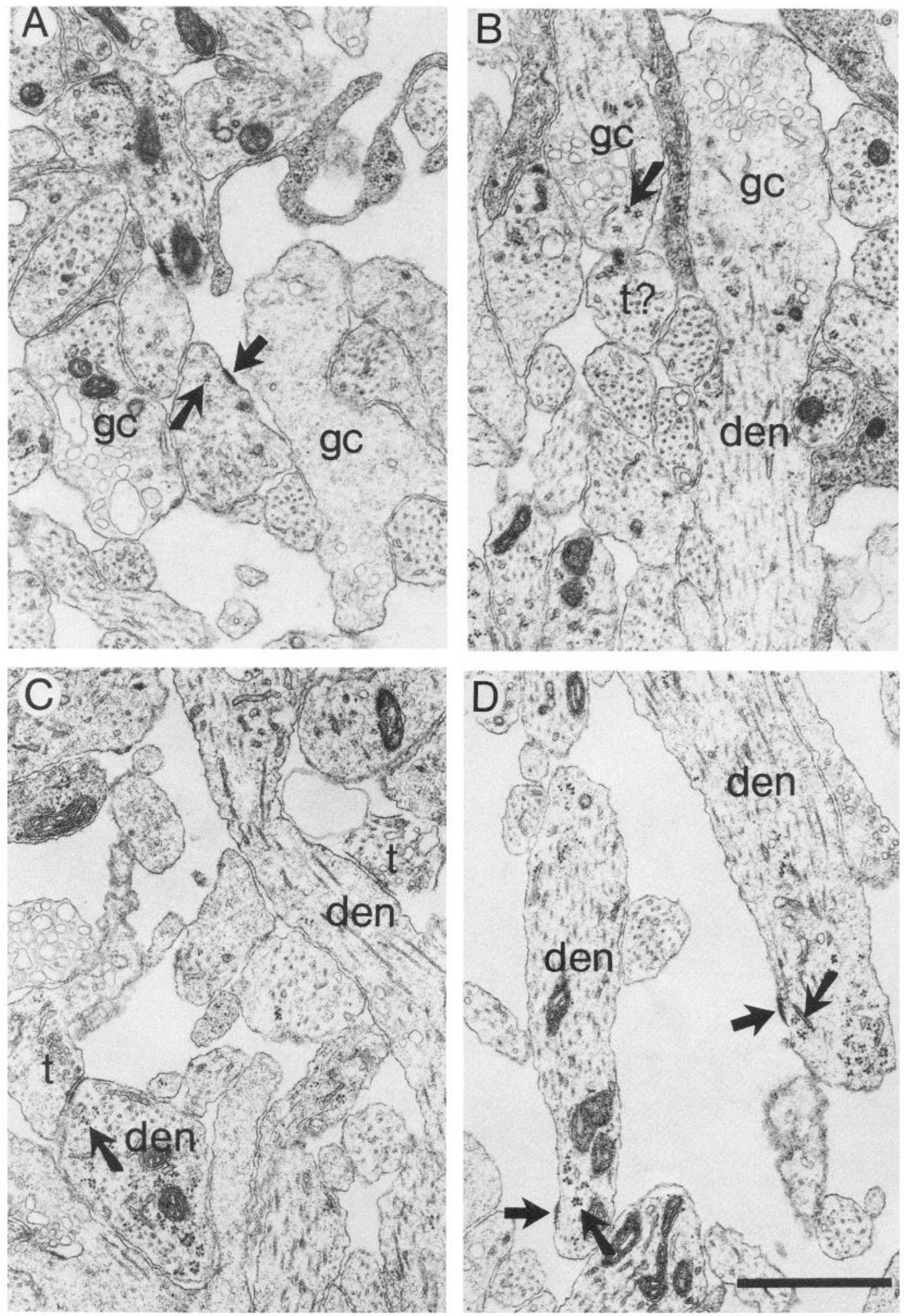

Figure 1. The molecular layer of the suprapyramidal blade of the dentate gyrus of $1 \mathrm{~d}$ old rats. A, Two types of profiles previously identified as growth cones $(\mathrm{gc})$ (i.e., enlarged profiles with vesicles resembling endoplasmic reticulum and filamentous enlargements with few other cytoplasmic specializations). Slanted arrow indicates polyribosomes under membranous densities resembling postsynaptic densities. Straight arrow indicates presumed empty psd's. $B$, Two vesicle-filled growth cones $(g c)$ with polyribosomes near but not within the growth cone proper. Dendrite (den), identified by presence of polyribosomes. Slanted arrow, Polyribosomes under what may be an immature synaptic contact (the presynaptic terminal is indicated by $t$ '). $C$, One of the rare mature-appearing synapses at $1 \mathrm{~d}$ of age. $D$, Two apparently noninnervated psd's with underlying polyribosomes. Calibration bar, $1 \mu \mathrm{m}$. 

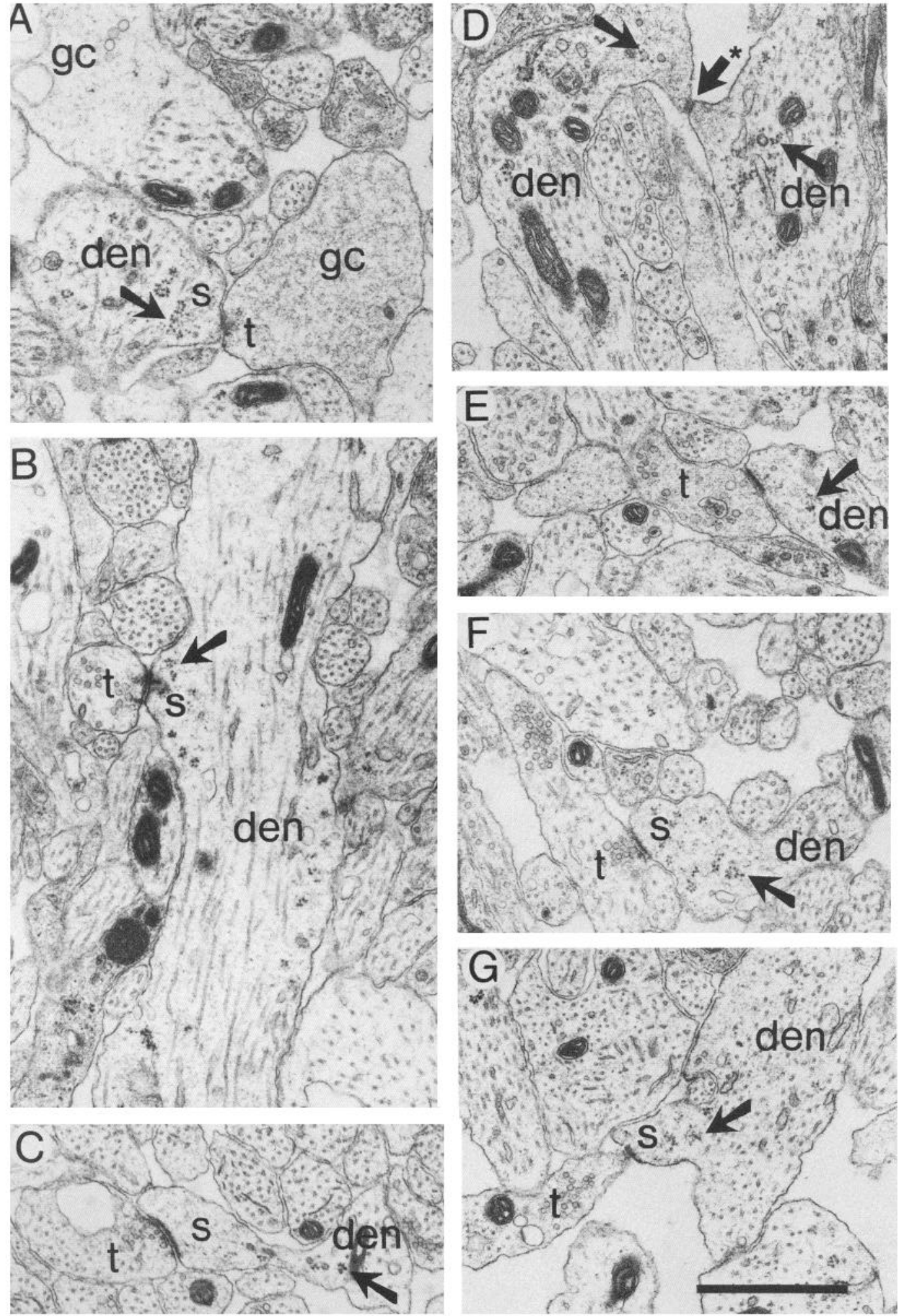

Figure 2. The molecular layer of the suprapyramidal blade of the dentate gyrus of $4 \mathrm{~d}$ old rats. A, An apparent spine in contact with a growth cone $(g c) . B$, The very stubby spines characteristic of this age. $C$, An example of one of the more rare elongated spines. The arrow with asterisk in $D$ indicates a dendrodendritic contact. $E-G$. Examples of spine synapses with polyribosomes. $s=$ spine; other symbols and abbreviations as for Figure 1. Calibration bar, $1 \mu \mathrm{m}$.

onal and dendritic processes, some of which had the characteristics of growth cones (Fig. 3, $A$ and $B$ ). Very few synapses were present, however, and those that were appeared quite immature, again containing few vesicles of the size of typical synaptic ves- icles, and often containing larger vesicles.

The suprapyramidal blade appeared more mature than at 4 $\mathrm{d}$ of age, with a larger number of synapses, and an increase in the apparent degree of differentiation of the synaptic contact 

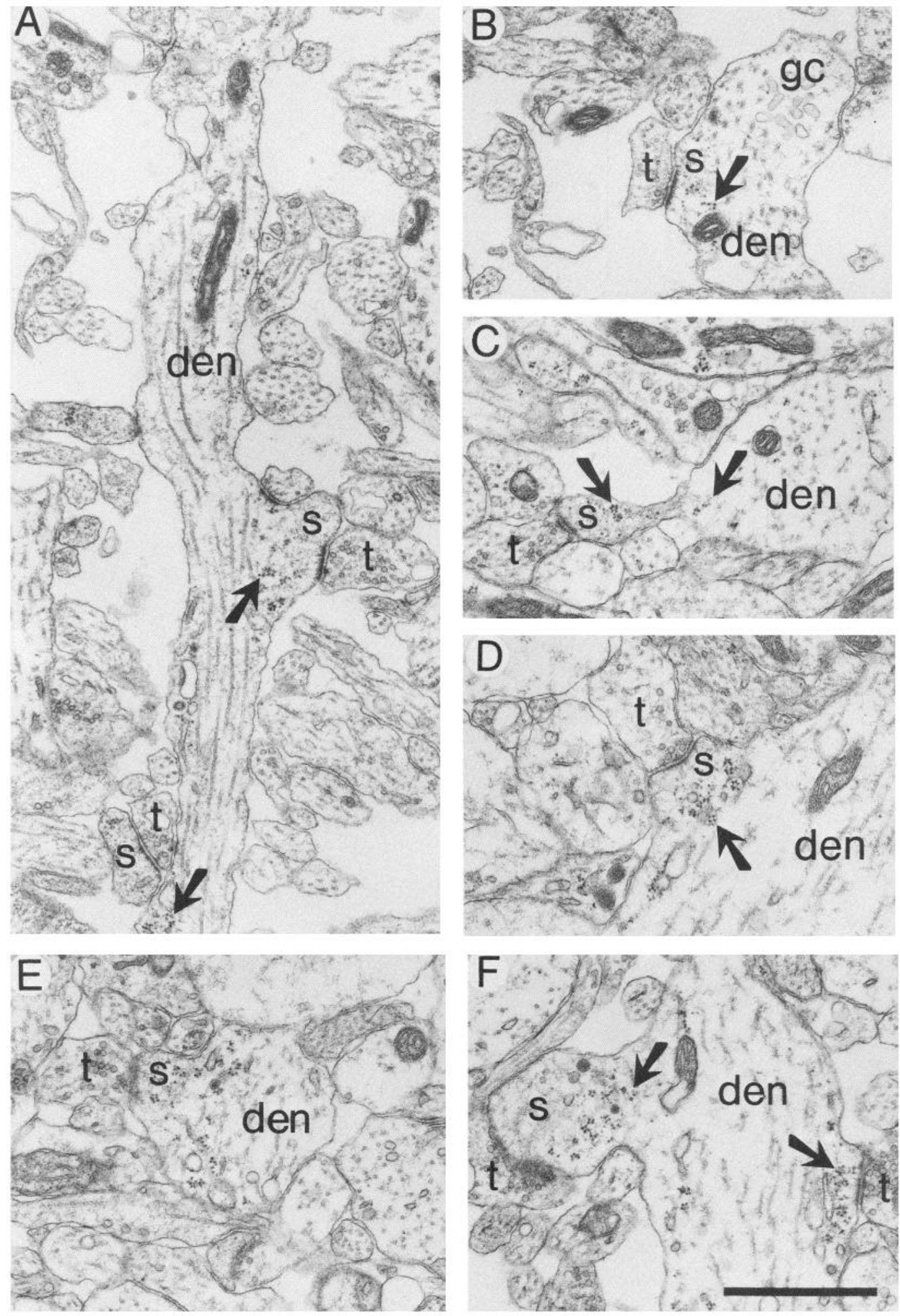

Figure 3. Examples of developing spine synapses from the molecular layer of the dentate gyrus at $7 \mathrm{~d}$ of age. $A$ and $B$ were taken in the infrapyramidal blade, while $C-E$ were taken in the suprapyramidal blade. Note the accumulations of polyribosomes under and within the spines, and the fact that the polyribosomes seem to be preferentially localized under the spines (see particularly $A$ ). Portions of the dendritic segments illustrated in $A$ and $B$ resemble growth cones, with large vesicles of smooth endoplasmic reticulum. Abbreviations and symbols as in Figure 1. Calibration bar, $1 \mu \mathrm{m}$.

region (Fig. 3, $C-F$ ). For example, many synaptic contacts were associated with profiles that were unambiguous spines, although most spines still appeared more stubby and less well-defined than in more mature animals. Only a few spines were found with narrow necks and enlarged heads (see Fig. $3 C$ for an example).

As is evident in Figure 3, $C-E$, accumulations of polyribosomes under the site of synaptic contact were particularly strik- 

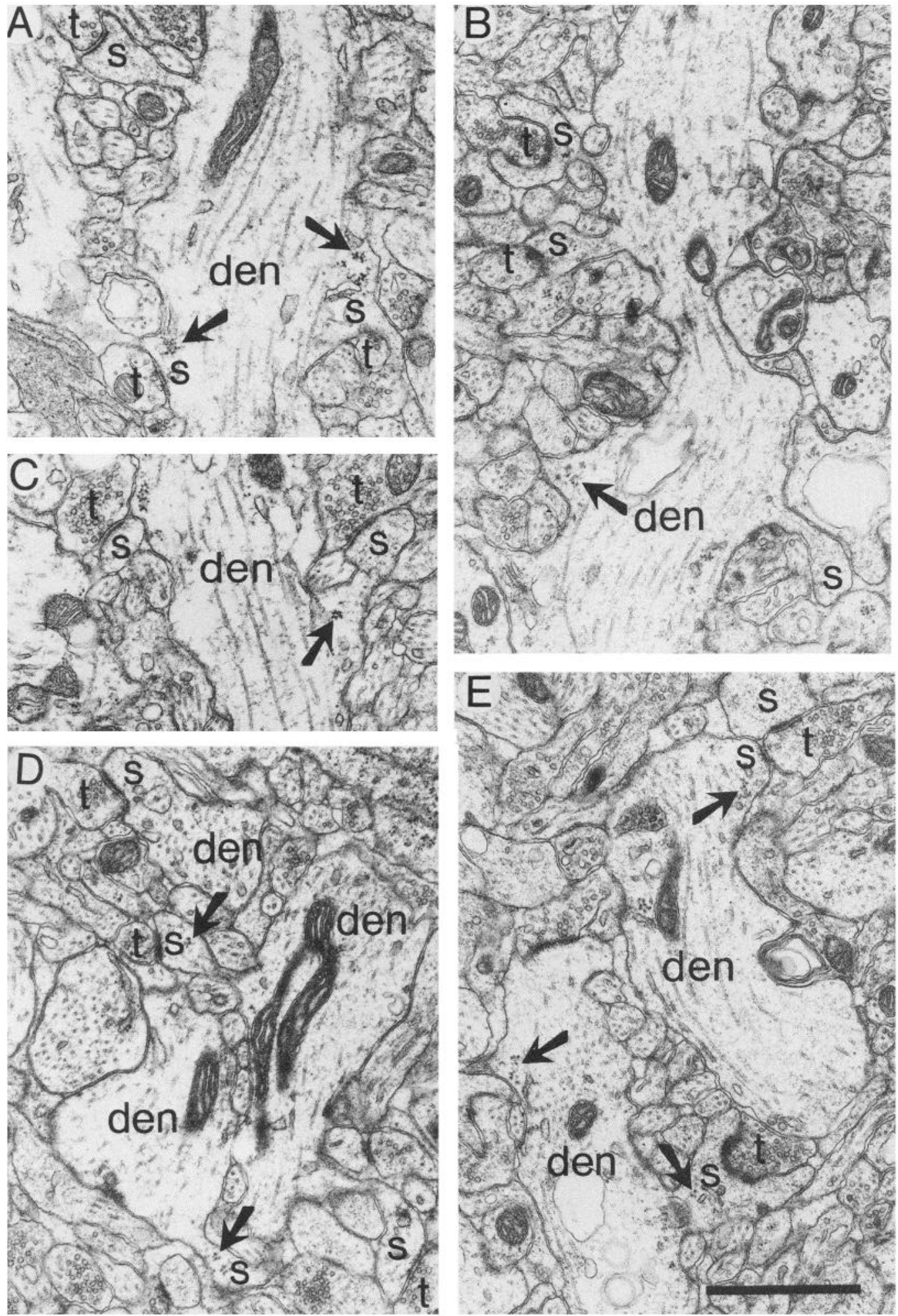

Figure 4. Developing spine synapses from the molecular layer of the dentate gyrus at $10(A$ and $B)$, and $15(C-E) \mathrm{d}$ of age. Note that the neuropil appears somewhat more mature, both in terms of numbers of synapses and in terms of the shape of the spines. Symbols and abbreviations as in Figure 1. Calibration bar, $1 \mu \mathrm{m}$.

ing in the $7 \mathrm{~d}$ old animals. Most of the polyribosomes appeared to lie within the stubby protrusion of the spine, out of the cylinder of the dendrite. The polyribosome clusters were both dramatic, with many well-defined rosettes under individual spines, and ubiquitous, in that most spines appeared to contain at least one cluster (see below for quantitative considerations). The selectivity of the localization of the polyribosomes under spines was still quite apparent (Fig. $3 A$ ).

The general picture of the molecular layer at $10 \mathrm{~d}$ of age was similar to that at $7 \mathrm{~d}$, except that synapses appeared to be some- 
what more mature (Fig. 4, $A$ and $B$ ), and even qualitatively it was clear that there were more of them. However, most spines were still quite stubby, and very few had the thin necks and large heads typical of mature spines. Polyribosomes remained prominent, but spines with multiple clusters of ribosomes were encountered less frequently.

By $15 \mathrm{~d}$ of age, the synapses appeared even more mature (Fig. $4, C-E$ ). Some spines had well-defined narrow necks and enlarged heads - the lollipop or mushroom shape characteristic of mature spines. The lollipop shape did not appear to be as well developed as in mature animals, however, in that many spines had necks almost as wide as the heads. Furthermore, most spines appeared to be shorter than those of mature animals. The clusters of polyribosomes under spines were less dramatic than at 7 and $10 \mathrm{~d}$ of age. Few profiles had the sorts of multiple rosettes that were characteristic of the younger ages, although, qualitatively, it was clear that the proportion of spines with polyribosomes was still higher than in mature animals. In addition, polyribosomes were common in the spine head, which is rarely seen in mature animals (Steward, 1983a).

The trends toward synapse maturation continued between 15 and $20 \mathrm{~d}$ of age. The spines in the neuropil of $20 \mathrm{~d}$ old animals appeared somewhat longer and more mature (in terms of the development of adult-like shape), although the neuropil was still easily distinguishable from the adult. By $28 \mathrm{~d}$ of age, however, the neuropil appeared qualitatively mature.

\section{Quantitative observations}

\section{Quantitative assessment of synaptogenesis}

Our quantitative evaluations focused on the suprapyramidal blade. As illustrated in Figure $5 A$, synapse density in the suprapyramidal blade was quite low at $1 \mathrm{~d}$ of age; indeed, an average of only 0.36 synapses were present per $100 \mu \mathrm{m}^{2}$ of neuropil. Synapse density increased in an almost linear fashion between 1 and $15 \mathrm{~d}$ of age, and continued to increase at a rate that was only slightly slower between 15 and $28 \mathrm{~d}$ of age. By 28 $\mathrm{d}$ of age, the average synapse density in the middle molecular layer of the suprapyramidal blade of the dentate gyrus was 49 synapses per $100 \mu \mathrm{m}^{2}$. This represents a 136-fold increase in synapse density between postnatal days 1 and 28 . Interestingly, synapse density was slightly higher at $28 \mathrm{~d}$ of age than in adult animals (see Fig. 5). The number of shaft synapses was quite low throughout the postnatal period (see Fig. $5 \mathrm{~A}$, broken line).

From the increase in synapse density across the postnatal period, the rate of synapse addition during each interval was calculated by determining the difference in synapse density between each age (how many new synapses were added) and dividing this number by the number of days in the interval. Thus, between 1 and $4 \mathrm{~d}$ of age, synapse density increased from 0.36 to 7.4 synapses $/ 100 \mu \mathrm{m}^{2}$ (7.1 synapses were added per $100 \mu \mathrm{m}^{2}$ in $3 \mathrm{~d}$ ). The rate of synapse addition was thus 2.4 synapses $/ 100$ $\mu \mathrm{m}^{2} / \mathrm{d}$. Similar calculations revealed rates of synapse addition of 2.2 synapses $/ 100 \mu \mathrm{m}^{2} / \mathrm{d}$ between 4 and $7 \mathrm{~d}, 2.0$ synapses $/ 100$ $\mu \mathrm{m}^{2} / \mathrm{d}$ between 7 and $10 \mathrm{~d}, 2.4$ synapses $/ 100 \mu \mathrm{m}^{2} / \mathrm{d}$ between 10 and $15 \mathrm{~d}, 1.4$ synapses $/ 100 \mu \mathrm{m}^{2} / \mathrm{d}$ between 15 and $20 \mathrm{~d}$, and 1.3 synapses $/ 100 \mu \mathrm{m}^{2} / \mathrm{d}$ between 20 and $28 \mathrm{~d}$. Thus the number of new synapses added per $100 \mu \mathrm{m}^{2}$ was remarkably constant over much of the developmental period.

\section{Increases in the thickness of the molecular layer}

Simple measures of synapse density do not provide a complete picture of synaptogenesis, since there is a considerable increase in the volume of the molecular layer over the postnatal interval. For the purposes of the present study, the principal concern was when synaptogenesis occurred; less important was the total extent of synaptogenesis. Nevertheless, volumetric increases could interact with measures of synapse density in such a way that a
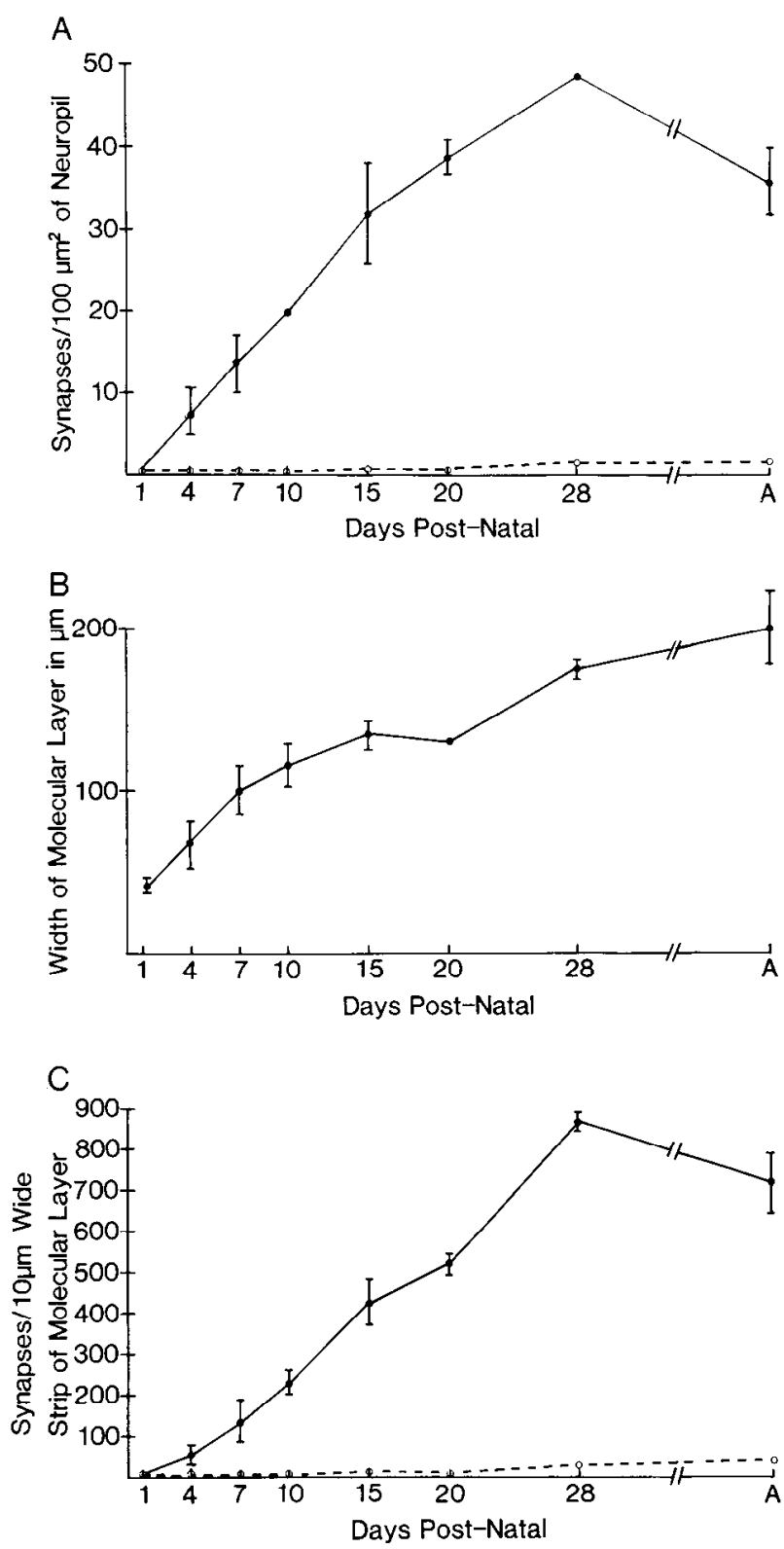

Figure 5. Synaptogenesis in the suprapyramidal blade of the dentate gyrus. $A$, Synapse density per unit area. Total spine synapses are indicated by the solid line and shaft synapses by the broken line. $B$, Thickness of the molecular layer of the suprapyramidal blade at the site from which counts of synapses were made. $C$, Estimated number of synapses per $10-\mu \mathrm{m}$-wide strip of the molecular layer (in order to estimate the overall extent of synaptogenesis, taking into account both the increases in synapse density per unit area and the increases in the volume of the neuropil). Vertical bars, The range of values at each age. $A$, Values from adult animals.

misleading picture would be presented regarding the time of most active synaptogenesis. For this reason, we attempted to obtain a more complete picture of the extent of the increase in synapses over the postnatal interval by correcting for increases in neuropil volume.

A rough indication of the increase in the neuropil can be obtained simply by measuring the thickness of the molecular layer of the suprapyramidal blade at the point of sampling. This is obviously not an accurate indication of the overall increase in the volume of the molecular layer, since there is an increase in septotemporal length, mediolateral breadth, and dorsoventral height. Nevertheless, volumetric measurements are not useful 


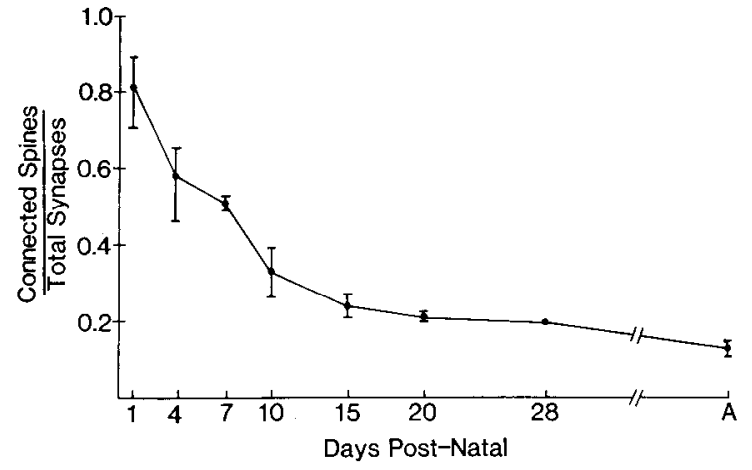

Figure 6. Proportion of the total number of observed synapses whose connection with the dendritic shaft can be seen in the plane of section. This ratio provides a quantitative but indirect measure of the increase in the length and decrease in diameter of dendritic spines, since the probability of finding a spine head in continuity with the dendritic shaft decreases as the spine length increases and spine neck diameter decreases. Vertical bars, The range of values at each age. $A$, Values from adult animals.

because of the gradients in maturation; unless one sampled several areas for estimates of synapse density, correcting values for total volumetric increases would be misleading. The increase in the height of the molecular layer provides an estimate of the volumetric increase at the sampling site, which can then aid in determining, at least roughly, what the actual increase in the number of synapses at that point in the neuropil must be. The measured thickness of the molecular layer in each animal (Fig. $5 B$ ) was used to determine the area (in $\mu \mathrm{m}^{2}$ ) of a $10-\mu \mathrm{m}$-wide strip from the top of the granule cell layer to the distal tips of the granule cell dendrites (the hippocampal fissure). We then multiplied the area thus obtained in each animal by the measured synapse density obtained from the same animal, providing an estimate of the total number of synapses in a $10-\mu \mathrm{m}$-wide strip across the molecular layer (see Fig. 5C). As is evident, correcting for the increases in the thickness of the molecular layer does not alter the conclusions regarding the time of most active synaptogenesis.

\section{A quantitative measure of spine elongation}

As was noted above, an aspect of maturation that was qualitatively obvious was an increase in spine length. In the youngest animals, synapses were found on blunt protrusions from the dendrite, whereas more and more mature-appearing spines were found as synaptogenesis proceeded. This increase in spine length was reflected by an increase in the proportion of synapses on spine heads that did not exhibit a continuity with a main dendritic shaft in the section plane (see Fig. 4). One way to obtain a quantitative indication of this spine elongation is to determine the proportion of synapses on "connected spines" (those in continuity with a dendritic shaft) across the postnatal interval (see Fig. 6). At $1 \mathrm{~d}$ of age, $80 \%$ of the synapses were on spines whose connection with the main dendritic shaft could be seen in the plane of section. The proportion of synapses on connected spines decreased over the postnatal interval, reaching a plateau of about $20 \%$ by $15 \mathrm{~d}$ of age, which was maintained through day 28 . There was an additional slight decrease in the proportion of connected spine heads after $28 \mathrm{~d}$ of age. These data provide a quantitative, but indirect, indication of the time course of elongation of dendritic spines across the postnatal period. However, it is likely that once spine length has reached a certain value, further increases would not affect this parameter. Thus, there could be a further elongation of spines after $15 \mathrm{~d}$ of age, which would not be reflected by this measure.
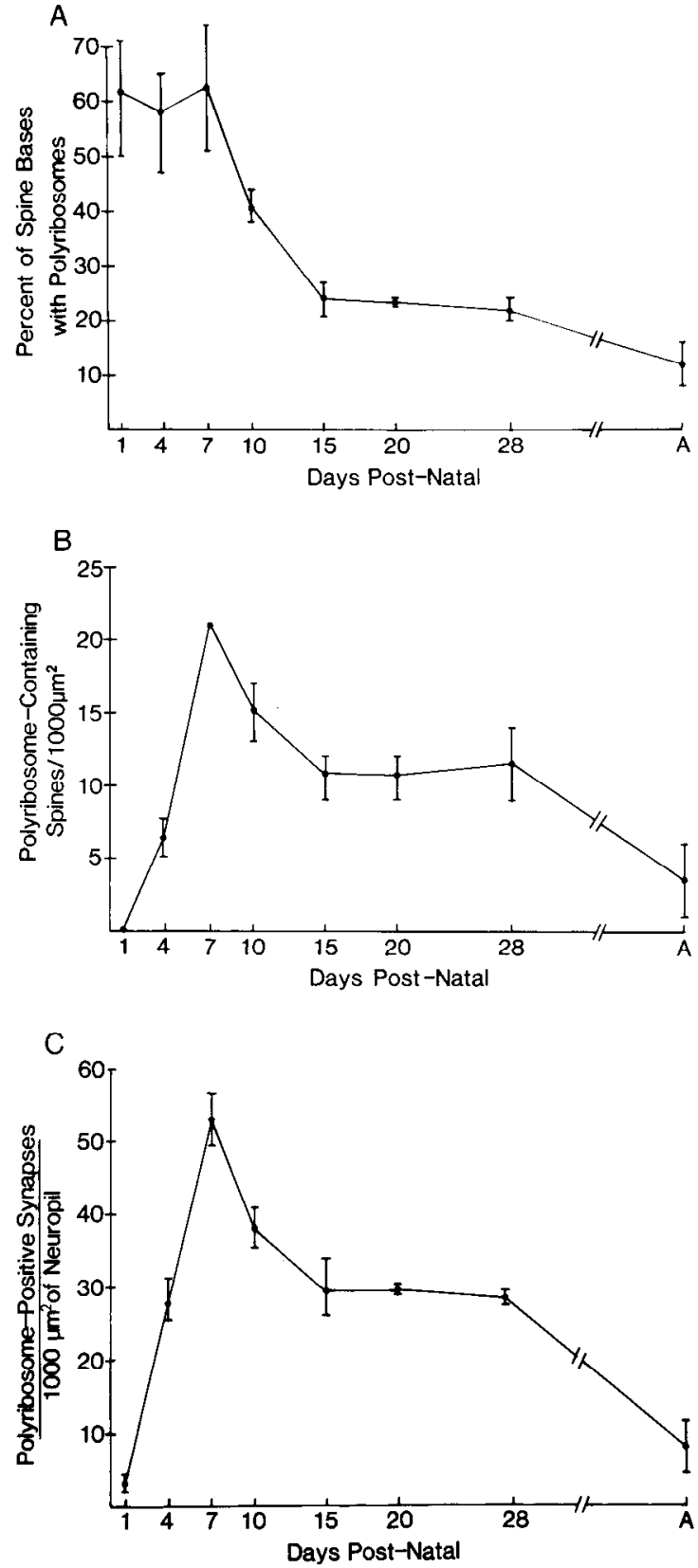

Figure 7. Three measures of the prominence of polyribosomes under synaptic contacts across the postnatal interval. $A$, Incidence of polyribosome-containing spine bases (the proportion of spine bases with underlying polyribosomes). $B$, Number of polyribosome-containing spines (spines with polyribosomes in either the head or neck region) per area of neuropil at each age. $C$, Total number of polyribosome-positive synapses per area of neuropil across the postnatal interval (spine synapses with polyribosomes at the base or within the spine head or neck, and shaft synapses with underlying polyribosomes). Vertical bars, The range of values. $A$, Values from adult animals.

\section{Quantitative analyses of polyribosomes associated with developing synapses}

Figure $7 A$ illustrates the incidence of polyribosomes under identified spines across the developmental period and in adult rats. As is evident, the proportion of spine synapses with underlying polyribosomes was highest at 1,4 , and $7 \mathrm{~d}$ of age; at all three ages, about $60 \%$ of the identified spine bases had underlying polyribosomes. The incidence of polyribosomes decreascd bctween 7 and $10 \mathrm{~d}$ of age, and again between 10 and $15 \mathrm{~d}$, and 
then remained at a plateau until $28 \mathrm{~d}$ of age. Interestingly, while synapse density had reached adult levels by $20 \mathrm{~d}$ of age, and was actually higher than adult levels at $28 \mathrm{~d}$ of age, the incidence of polyribosomes at 20 and $28 \mathrm{~d}$ of age was considerably higher than in mature animals. Thus, the incidence of polyribosomecontaining spines was highest when most of the synapses were presumably newly formed or under construction, but was also higher than adult levels throughout the period when synapse numbers were being adjusted.

In our previous study of synapses growing in response to lesions in adult animals (Steward, 1983a), the increases in polyribosomes under spine bases were accompanied by increases in polyribosomes within spine heads and necks, suggesting a movement of the protein-synthetic machinery out into the spine proper during synapse construction. This sort of analysis was complicated in the present study by the fact that spines were quite short in the very young animals; thus, distinguishing the point of transition between the dendritic shaft and the spine was difficult. Nevertheless, when polyribosomes that appeared to lie within the spine proper (either within the head or the neck) were separately counted, there was a clear peak in the number of polyribosome-containing spines per area of neuropil at $7 \mathrm{~d}$ of age (see Fig. $7 B$ ). These results must be interpreted cautiously, since polyribosomes at the base of the spine would lie much closer to the synaptic site in short spines even if there were no migration out into the spine proper. Thus, certain sectioning angles might yield profiles in which the polyribosomes appeared to lie within a spine head even though the polyribosomes were actually still at the intersection between the spine base and the dendritic shaft. Even if the polyribosomes do migrate out into the growing spine, the actual distance of the polyribosomes from the main shaft of the dendrite is not great in short spines. Nevertheless, these results provide additional evidence for a special relationship between the polyribosomes and the synaptic site during synapse construction.

We were very interested in determining whether polyribosomes were also present under shaft synapses during the developmental period. To address this question, we adopted a very rigorous criterion for shaft synapses. $\Lambda$ s was noted above, many early forming synapses were found on very blunt protrusions from dendrites. These were classified as spines for quantitative purposes whenever there was even the slightest indication of a protrusion at the site of the postsynaptic specialization, or at either side. Synapses were classified as shafts only if the profile of the dendrite was entirely flat at and around the synaptic region. Thus, while some of the synapses classified as spines might actually be shaft synapses, the opposite error is minimized. Because of this rigorous criterion, very few shaft synapses were identified throughout the developmental period (see Fig. $5 A$, broken line); nevertheless, the same trends seemed to apply regarding the higher incidence of polyribosomes during synapse formation. For example, combining the data from all 1, 4, and $7 \mathrm{~d}$ old animals yielded a total of 50 shaft synapses; 16 of these $(32 \%)$ had underlying polyribosomes. A total of 71 shaft synapses were found in the $10-15 \mathrm{~d}$ old animals; 11 of these $(15 \%)$ had underlying polyribosomes. Finally, a total of 80 shaft synapses were found in the animals sacrificed at 20-28 d of age; five of these $(6 \%)$ had underlying polyribosomes.

If the proportion of synapses with polyribosomes remains constant between 1 and $7 \mathrm{~d}$ of age while the number of synapses increases, there must be an increase in the total number of synapses with polyribosomes per area of neuropil during this interval. This is of interest, since the impression gained from qualitative observation was that the most dramatic accumulations of polyribosomes were at $7 \mathrm{~d}$ of age. To directly determine the total number of synapses with associated polyribosomes per area of neuropil, we determined the number of polyribosomecontaining spine bases and spine heads, as well as shaft synapses with polyribosomes in the area of neuropil sampled. As illustrated in Figure $7 C$, the number of polyribosome-positive synapses per area of neuropil was negligible at $1 \mathrm{~d}$ of age, despite the fact that the majority of the synapses present had polyribosomes. The number of polyribosome-positive synapses increased dramatically after day 1 , reached a peak at $7 \mathrm{~d}$ postnatal, and subsequently decreased to a plateau that was maintained between 15 and $28 \mathrm{~d}$ of age. Again, there was a further decrease in this measure between $28 \mathrm{~d}$ and adulthood.

\section{Discussion}

The present results strongly support our previous suggestion, based on studies of synapse growth following lesions, that polyribosomes are particularly prominent at the postsynaptic site during synaptogenesis (Steward, 1983a, b; Steward and Falk, 1985; Steward and Fass, 1983; Steward, in press). Prior to discussing the possible role of the polyribosomes, it is necessary to briefly discuss the quantitative methods upon which the conclusions are based.

It is, first, important to note that the measures of synapse density that are herein presented are not necessarily representative of synapse density throughout the dentate gyrus; the gradient in maturation between supra- and infrapyramidal blades makes it difficult or impossible to obtain an overall picture (for a discussion of this problem, see Cowan et al., 1980). For example, synaptogenesis in the infrapyramidal blade is clearly several days behind that in the suprapyramidal blade (compare the present data with that of Crain et al., 1973). The fact that the data may not be representative for the cntire dentate gyrus does not affect our conclusions, since the key issue is the relationship between the time course of synaptogenesis at the sampling site and the prevalence of polyribosomes in the synapses that are found at that site.

A sccond limitation that should be recalled has been discussed in previous studies: The quantitative analyses we have utilized provide only a relative measure of the incidence of polyribosomes under spines (Steward, 1983a; Steward and Falk, 1985; Steward and Levy, 1982). Even with the smallest spines, a single thin-section does not reveal all of the area comprising the spine base; thus, some of the spines that do not have polyribosomes in a given section nevertheless have underlying polyribosomes that can be revealed by a serial section reconstruction of the entire spine base (see Steward, 1983a; Steward and Levy, 1982). For this reason, measures of the incidence based on single sections (what we have termed "relative incidence") consistently underestimate the actual incidence. The extent of the underestimate in the developing animals is not known; particularly in the youngest animals, spines are stubby, and their bases subsume a large area. At the earliest ages, it may well be that every spine has underlying polyribosomes.

While the quantitative measurements have limitations, we do not feel that these limitations compromise in any way the conclusions that the proportion of synapses with polyribosomes is highest at $1-7 \mathrm{~d}$, during the initial stages of synaptogenesis, whereas the number of polyribosome-containing synapses/area of neuropil is greatest at about $7 \mathrm{~d}$. A discussion of the significance of these observations requires a consideration of the other events taking place in the dentate gyrus at this time.

\section{The development of the dentate gyrus}

A fairly complete picture is now available regarding many aspects of the development of the dentate gyrus (for a review, see Cowan et al., 1980). These data can be reviewed only very briefly here. Granule cells of the dentate gyrus are generated over a prolonged period from embryonic day 14 through about the second postnatal week (Bayer, 1980; Schlessinger et al., 1975). While the infrapyramidal blade is developmentally much less mature than the suprapyramidal blade throughout early devel- 
opment, there is a continuing addition of cells to both blades (Bayer, 1980). This means that even though the neuropil of the suprapyramidal blade is well developed by $7-10 \mathrm{~d}$, a substantial number of granule cells are just beginning to grow dendrites into the neuropil layer. Interestingly, the peak of neurogenesis occurs toward the end of the first postnatal week (days 5-8; see Schlessinger et al., 1975). This is at about the same time that the greatest number of polyribosome-containing synapses are found.

Only limited information is available regarding the development of the granule cell dendrites. The existing data, based on Golgi-stained material, suggest that the granule cells elaborate their characteristic dendritic trees between birth and $20 \mathrm{~d}$ of age (Fricke, 1975; reviewed in Cowan et al., 1980). There was no distinction between the supra- and infrapyramidal blades in the quantitative summary of increases in dendritic length over the developmental period. Given the substantial differences in the maturation of supra- and infrapyramidal blades, the combination of data from both blades makes it impossible to relate the available Golgi data to the present electron-microscopic evidence, except to say that dendritic growth seems to occur over roughly the same period as the increases in synapse density.

Despite the relative immaturity of the dentatc gyrus at birth, at least some of the afferents appear in the neuropil very early: fibers of the dentate commissural system can first be detected in the neuropil the day after birth (Cowan et al., 1980), and the projection from the entorhinal cortex appears to develop somewhat earlier (Fricke and Cowan, 1977; Loy et al., 1977). The presence of these projections at an early period suggests that at least some presynaptic fibers are present before very much dendritic growth has occurred, and long before the peak in polyribosome-containing spines.

Because synaptogenesis is ongoing while some granule cells are just beginning to grow dendrites into the molecular layer, the age of given dendrites cannot be determined, and any estimation of the probable age of given synaptic contacts is quite questionable. Presumably, very immature synapses coexist with ones that are several days old. Nevertheless, if synapse density increases over days, and if there is no compression of the neuropil during the same interval, then the increase in synapse density indicates the number of synapses added. In fact, given that there is a simultaneous growth of the neuropil, the increase in synapse density provides a minimum estimate of the number of new synapses in a given area of neuropil. It is therefore probably safe to conclude that, between 1 and $15 \mathrm{~d}$ of age, each $100 \mu \mathrm{m}^{2}$ of neuropil contains at least $2-2.5$ synapses that have been formed within the preceding $24 \mathrm{hr}$.

\section{Possible role of the polyribosomes}

The present data do not by themselves reveal what role the spine-associated polyribosomes play; they do, however, permit more informed speculation than has heretofore been possible. $A$ priori, the participation of the polyribosomes in dendritic elongation seems unlikely; they are associated with synapses, and are essentially absent from dendritic growth cones. Thus, attention is focused on the synaptic site. Synapse formation during development involves a number of sequential processes, beginning with the growth of the axon and dendrite into apposition, and ending with the elaboration of all of the structural specializations of the contact region, including the postsynaptic membrane density and the microspecialization of form (the spine). The polyribosomes and the proteins they produce could be involved in (1) inducing or promoting the growth of the axon or attracting it to the postsynaptic site; (2) the formation of the initial contact (i.e., recognition or adhesion); or (3) the differentiation of the mature synaptic contact. The latter is by far the broadest of the possibilities, involving the construction of the contact region itself (i.e., the production of receptors, ion channels, structural proteins of the postsynaptic membrane specialization, proteins involved in the stabilization of the membrane proteins, or any of a number of enzymes that are found at the postsynaptic site, such as calmodulin or protein kinase); the building of the dendritic spine, and perhaps the elaboration of molecules that induce or facilitate some aspect of this final differentiation, and which, themselves, are not part of the synaptic contact.

The hypothesis that the proteins produced by the polyribosomes promote the growth of the axon, or attract it to the contact site, was initially proposed because the increases in polyribosomes following lesions occurred at the initial phase of the presynaptic growth response, prior to the time of significant axonal ingrowth (Steward, 1983a). This hypothesis would therefore predict that axon ingrowth ought to be associated with the peak in polyribosomes at $7 \mathrm{~d}$ of age. We did not measure the rate of ingrowth of presynaptic processes, since these could not be identified with certainty until they formed contacts; however, the constant rate of synapse addition over the postnatal interval would suggest a constant rate of axon ingrowth. In addition, there is ample evidence for a substantial availability of afferents even at $1 \mathrm{~d}$ of age (for a review, see Cowan et al., 1980). Thus, the present data argue against the possibility that the polyribosomes produce a factor to promote axon ingrowth.

If the polyribosomes were set out by the postsynaptic cell to induce or promote initial contact formation, one would also expect the number of polyribosome-positive spines to be closely related to the number of recently formed contacts. However, the rate of synapse addition appears to be constant from 1 to $15 \mathrm{~d}$ of age (averaging about two synapses added per $100 \mu \mathrm{m}^{2} /$ d), whereas the number of polyribosome-positive synapses is greatest at $7 \mathrm{~d}$ of age. Given the problems with asynchronous growth of the granule cells during the postnatal interval, these observations certainly do not rule out the possibility that polyribosomes are set out by the postsynaptic neuron to induce or promote contact formation, but the observations are not consistent with the predictions of the hypothesis either.

The hypothesis that the polyribosomes produce proteins involved in the growth of the dendritic spine is not supported, since it would predict that polyribosomes would not be present at nonspine synapses. While the data in this regard are not compelling, owing to the small number of shaft synapses in the molecular layer of the dentate gyrus, polyribosomes were more prominent under the shaft synapses found during the period that they were most prominent under spines. In addition, we have recently found that polyribosomes are associated with nonspine synapses on axon initial segments (O. Steward and C. E. Ribak, unpublished observations). These results do not disprove the hypothesis that the polyribosomes produce proteins related to the construction of the spine, but they do make the alternative possibilities more attractive.

We are left with the hypothesis that the polyribosomes and the proteins that they produce contribute to the differentiation of the synapse; the protein(s) could be a component of the synaptic region itself, or could be necessary for differentiation of the synapse, but not be a component (i.e., some retrograde factor that might stabilize the presynaptic terminal; see Changeux and Danchin, 1976). The construction of mature synapses can be viewed as a series of stages, with each stage representing a decision point at which the construction of the synapse can either be continued or aborted (see Burry, 1982). For example, it has been suggested that synapses initially are labile, and progress to stable contacts as a consequence of functional interactions with the target (see Changeux and Danchin, 1976). The duration of the labile phase could depend on a number of factors and need not come at a specified time after the initial contact. Thus, the peak in synapse-associated polyribosomes at $7 \mathrm{~d}$ could herald 
a stage of synapse maturation; perhaps this stage is brought about by some change in the functional activity of either preor postsynaptic elements, or perhaps it is related to more general effects (hormones, or experience). In this regard, it is of considerable interest that there is an increase in polyribosome-containing spine synapses in the visual cortex in animals that had been exposed to an enriched environment during development (Greenough et al., 1985).

As we noted previously (Steward and Falk, 1985), the fact that polyribosomes are also present under spine synapses in mature animals does not contradict the suggestion that they are involved in synapse growth. There is a growing consensus that there is a natural turnover of synapses in mature animals, which may be particularly important for replacing or repairing synapses damaged by injury or disease (Cotman et al., 1981). Furthermore, it has long been suspected that the storage of experiential information may also require synapse growth (Tanzi, 1893; and see Cotman et al., 1981). If polyribosomes under the synapse are a hallmark of growing synapses, and if there is normally an ongoing synapse turnover in mature animals, it is possible that polyribosome-containing spines in mature animals mark synapses under construction. If this is true, a much needed means would be available for identifying growing synapses in mature nervous systems.

\section{References}

Bayer, S. A. (1980) Development of the hippocampal region in the rat: I. Neurogenesis examined with $3 \mathrm{H}$-thymidine autoradiography. J. Comp. Neurol. 190: 87-114.

Bloom, F. E. (1972) The formation of synaptic junctions in developing rat brain. In Structure and Function of Synapses, G. D. Pappas and D. P. Purpura, eds., pp. 101-120, Raven, New York.

Burry, R. W. (1982) Development of apparent presynaptic elements formed in response to polylysine coated surfaces. Brain Res. 247: 116.

Carlin, R. K., D. J. Grab, and P. Siekevitz (1981) Function of calmodulin in postsynaptic densities III. Calmodulin-binding proteins of the postsynaptic density. J. Cell Biol. 89: 449-455.

Changeux, J.-P., and A. Danchin (1976) Selective stabilisation of developing synapses as a mechanism for the specificity of neuronal networks. Nature 264: 705-712.

Cohen, R. S., F. Blomberg, K. Berzins, and P. Siekevitz (1977) The structure of postsynaptic densities isolated from dog cerebral cortex I. Overall morphology and protein composition. J. Cell Biol. 74: 181203.

Cotman, C. W., M. Nieto-Sampedro, and E. W. Harris (1981) Synapse replacement in the nervous system of adult vertebrates. Physiol. Rev. 61: 684-784.

Cowan, W. M., B. B. Stanfield, and K. Kishi (1980) The development of the dentate gyrus. In Current Topics in Developmental Biology, 15: Neural Development, Part 1: Emergence of Specificity in Neural Histogenesis, pp. 103-157, Academic, New York.

Crain, B. J., C. Cotman, D. Taylor, and G. Lynch (1973) A quantitative electron microscopic study of synaptogenesis in the dentate gyrus of the rat. Brain Res. 63: 195-204.

Del Cerro, M. P., and R. S. Snider (1968) Studies on the developing cerebellum. Ultrastructure of the growth cones. J. Comp. Neurol. 133: 241-362.

Feit, H., P. Kelly, and C. W. Cotman (1977) Identification of a protein related to tubulin in the postsynaptic density. Proc. Natl. Acad. Sci. USA 74: 1047-1051.

Foelix, R. F., and R. Oppenheim (1974) The development of synapses in the cerebellar cortex of the chick embryo. J. Neurocytol. 3: 277294.

Fricke, R. (1975) Studies on the morphology and development of the hippocampus and dentate gyrus. Unpublished doctoral dissertation, Washington University, St. Louis, MO.

Fricke, R., and W. M. Cowan (1977) An autoradiographic study of the development of the entorhinal and commissural afferents to the dentate gyrus of the rat. J. Comp. Neurol. 173: 231-250.
Grab, D. J., R. K. Carlin, and P. Siekevitz (1981a) Function of calmodulin in postsynaptic densities I. Presence of a calmodulin-activatable cyclic nucleotide phosphodiesterase activity. J. Cell Biol. 89: 433-439.

Grab, D. J., R. K. Carlin, and P. Siekevitz (1981b) Function of calmodulin in postsynaptic densities II. Presence of a calmodulin-activatable protein kinase activity. J. Cell Biol. 89: 440-448.

Greenough, W. T., H.-N. S. Hwang, and C. Gorman (1985) Evidence for active synapse formation or altered postsynaptic metabolism in visual cortex of rats reared in complex environments. Proc. Natl. Acad. Sci. USA 82: 4549-4552.

Hillman, D. E. (1979) Neuronal shape parameters and substructures as a basis of neuronal form. In The Neurosciences Fourth Study Program, F. O. Schmitt and F. G. Worden, eds., pp. 477-498, M.I.T., Cambridge, MA.

Hinds, J. W., and P. L. Hinds (1976) Synapse formation in the mouse olfactory bulb. II. Morphogenesis. J. Comp. Neurol. 169: 41-62.

Kawana, E., C. Sandri, and K. Akert (1971) Ultrastructure of growth cones in the cerebellar cortex of the neonatal rat and cat. Z. Zellforsch. 115: 284-298.

Kelly, P. R., and C. W. Cotman (1978) Synaptic proteins: Characterization of tubulin and actin and identification of a distinct postsynaptic density polypeptide. J. Cell Biol. 79: 173-183.

Loy, R., G. Lynch, and C. W. Cotman (1977) Development of afferent lamination in the fascia dentata of the rat. Brain Res. 121: 229-243.

Pfenninger, K. H., and R. P. Rees (1976) From the growth cone to the synapse: Properties of membranes involved in synapse formation. In Neuronal Recognition, S. H. Barondes, ed., pp. 131-178, Chapman and Hall, London.

Schlessinger, A. R., W. M. Cowan, and D. I. Gottlieb (1975) An autoradiographic study of the time of origin and the pattern of granule cell migration in the dentate gyrus of the rat. J. Comp. Neurol. 159: 149-176.

Skoff, R. P., and V. Hamburger (1974) Fine structure of dendritic and axonal growth cones in embryonic chick spinal cord. J. Comp. Neurol. 153: $107-148$

Steward, O. (1983a) Alterations in polyribosomes associated with dendritic spines during the reinnervation of the dentate gyrus of the adult rat. J. Neurosci. 3: 177-188.

Steward, O. (1983b) Polyribosomes at the base of dendritic spines of central nervous system neurons: Their possible role in synapse construction and modification. Cold Spring Harbor Symp. Quant. Biol 48: $745-759$.

Steward, O. (in press) Protein synthesis under dendritic spine synapses during lesion-induced synaptogenesis: Evidence for regulation of reinnervation by the target cell. Exp. Brain Res. (Suppl.).

Steward, O., and P. M. Falk (1985) Polyribosomes under developing spine synapses: Growth specializations of dendrites at sites of synaptogenesis. J. Neurosci. Res. 13: 75-88.

Steward, O., and B. Fass (1983) Polyribosomes associated with dendritic spines in the denervated dentate gyrus: Evidence for local regulation of protein synthesis during rcinncrvation. In Molecular Interactions Underlying Higher Brain Functions, J.-P. Changeux, J Glowinski, M. Imbert, and F. E. Bloom, eds., pp. 131-136, Prog. Brain Res. 58.

Steward, O., and W. B. Levy (1982) Preferential localization of polyribosomes under the base of dendritic spines in granule cells of the dentate gyrus. J. Neurosci. 2: 284-291.

Steward, O., and S. V. Vinsant (1983) The process of reinnervation in the dentate gyrus of the adult rat: A quantitative electron microscopic analysis of terminal proliferation and reactive synaptogenesis. J. Comp. Neurol. 214: 370-386.

Tanzi, E. (1893) I Fatti i le induzioni nell'odierna istologia del sistema nervoso. Riv. Sperim. Freniatria Medic. Leg. 19: 419-472.

Tennyson, V. M. (1970) The fine structure of the axon and growth cone of the dorsal root neuroblast of the rabbit embryo. J. Cell Biol. 44: 62-79.

Vaughn, J. E., and T. J. Sims (1978) Axonal growth cones and developing axonal collaterals form synaptic junctions in embryonic mouse spinal cord. J. Neurocytol. 7: 337-363. 